\title{
Programa de acondicionamiento físico en pacientes con diálisis peritoneal: un estudio piloto
}

\author{
Jorge F. Yanguma-Valentín ${ }^{1 *}$, Adriana Campos-Rodríguez², Astolfo Romero-García ${ }^{2,3}$ y \\ Carlos A. Tobón- Reyes ${ }^{4}$
}

${ }^{1}$ Centro de Estudios en Medición de la Actividad Física (CEMA), Escuela de Medicina y Ciencias de la Salud, Universidad del Rosario; ${ }^{2}$ Grupo de Investigación en Ciencias Aplicadas a la Actividad Física, Deporte y la Salud (GICAEDS), Facultad de Cultura Física, Deporte y Recreación, Universidad Santo Tomás; ${ }^{3}$ Colegio Colombiano de Educadores Físicos y Profesiones Afines (COLEF); ${ }^{4}$ Fresenius Medical Care Colombia S.A. Bogotá, Colombia

\section{Resumen}

Antecedentes: Este estudio determinó el efecto de un programa de acondicionamiento físico sobre los indicadores bioquímicos, capacidad funcional y calidad de vida relacionados con la salud de pacientes con DP. Materiales y métodos: Estudio piloto con análisis por protocolo de los datos obtenidos. La población estuvo conformada por un grupo de 11 pacientes con DP de la Clínica NephroCare el Dorado Fresenius Medical Care Colombia S.A., Bogotá. La intervención tuvo una duración de 18 semanas. Las pruebas utilizadas fueron: SF-36, 6MWT, Handgrip, Sit to Stand, Sit and Reach, número de pasos diarios, peso, talla, IMC, \% graso, \% magro, sobrehidratación y parámetros bioquímicos. Resultados: Cinco pacientes (45.4\%) cumplieron con la totalidad (100\%) de la intervención y el resto no culminó precisamente por la complejidad de la ERC. En la capacidad funcional se evidenció el incremento significativo del número de repeticiones en el 30 Sit to Stand $(p=0.004)$; en la calidad de vida, la variable salud general mejoró en grado significativo $(p=0.044)$. Conclusiones: Se observó que un programa de acondicionamiento físico contribuye al aumento significativo en diferentes dominios de la calidad de vida, como función física, dolor corporal y vitalidad evaluados a través del SF-36 en los pacientes con DP.

Palabras clave: Enfermedad renal crónica. Diálisis peritoneal. Acondicionamiento físico. Calidad de vida. Capacidad funcional. Indicadores bioquímicos.

\section{Physical conditioning program in patients of peritoneal dialysis: a pilot study}

\section{Abstract}

Background: This study aimed to determine the effect of a physical conditioning program on biochemical indicators, functional capacity and health-related quality of life of $P D$ patients. Materials and methods: Pilot study with protocol analysis of the data obtained. The population consisted of a group of eleven patients with CKD in PD of the Dialysis Centre El Dorado Fresenius Medical Care Colombia. The intervention lasted 18 weeks. The tests used were: SF-36, 6MWT, Handgrip, 30 Sit to Stand, Sit and Reach, number of daily steps, weight, height, Body Mass Index, \% fat, \% lean, overhydration and biochemical parameters. Results: Five patients (45.4\%) complied with one hundred percent (100\%) of the intervention, the rest did not culminate precisely because of the complexity of the CKD. In the functional capacity the significant increase in the number

\section{Correspondencia:}

*Jorge F. Yanguma-Valentín

E-mail: joryan83@gmail.com
Disponible en internet: 18-06-2021

Nefro Latinoam. 2021;18:15-25 www.nefrologialatinoamericana.com

2444-9032/@ 2021 Sociedad Latinoamericana de Nefrología e Hipertensión. Publicado por Permanyer. Este es un artículo open access bajo la licencia CC BY-NC-ND (http://creativecommons.org/licenses/by-nc-nd/4.0/). 
Nefro Latinoam. 2021;18

of repetitions in the Sit to Stand was evidenced ( $p=0.004)$; in the quality of life the variable General Health improved significantly ( $p=0.044$ ). Conclusions: It was demonstrated that a program of physical conditioning, contributes to the significant increase in different domains of the quality of life as physical functioning, bodily pain, vitality evaluated through the SF-36 in $P D$ patients.

Key words: Chronic renal disease. Peritoneal dialysis. Exercise. Quality of life. Functional capacity. Biochemical indicators.

\section{Introducción}

En la actualidad, alrededor de 3 millones de pacientes con ERC se encuentran en tratamiento de reemplazo (TRR) en el mundo, cifra que va en aumento ${ }^{1}$. De éstos, 275000 pacientes equivalentes al 11\% están en diálisis peritoneal (DP) y los restantes en hemodiálisis (HD), considerada ésta como la TRR dominante ${ }^{2}$. Colombia no es la excepción y se mantiene esta tendencia antes expuesta, ya que según la Cuenta de Alto Costo del 2017 eran 7,274 pacientes en DP, lo que representa el $21 \%$. De manera adicional se observa con gran preocupación que cada vez son más "jóvenes" las personas que requieren TRR, es decir, pacientes con una media de 55 años de edad, con una desviación estándar de 16.4 años $^{3}$.

Asimismo, se ha podido observar que además de los indicadores bioquímicos, también compromete la capacidad funcional y la calidad de vida relacionada con la salud en los pacientes con enfermedad renal crónica (ERC), sobre todo en aquéllos sometidos a tratamiento de reemplazo renal, debido no sólo a la evolución agresiva sino a las complicaciones de esta anomalía ${ }^{4}$ y a su larga duración.

Es por esto que, cuando se analiza el comportamiento cinético de estos pacientes, se encuentra un compromiso primario de la función cardiovascular, respiratoria y muscular por desnutrición, inflamación y anemia ${ }^{5}$. Esto lleva al paciente de forma temprana a un estado de fragilidad ${ }^{6}$ con disminución de la condición física (capacidad funcional) ${ }^{7}$, una conducta sedentaria que orilla a la inactividad física ${ }^{8}$, mayor riesgo de sufrir enfermedades relacionadas con este tipo de población y aumento de los índices de morbimortalidad; todo ello produce un efecto negativo en los indicadores de salud pública ${ }^{9,10}$ e incide en el detrimento de la calidad de vida ${ }^{11}$.

Es por esta razón que se han llevado a cabo investigaciones para identificar los beneficios que representan para el paciente la inclusión y la participación en programas de acondicionamiento físico en esta población. En los últimos 10 años se han determinado los beneficios del acondicionamiento físico en relación con el incremento de la capacidad aeróbica ${ }^{12}$, la estructura muscular ${ }^{13}$ y la función neuromuscular ${ }^{14}$ que al final mejoran la funcionalidad ${ }^{15}$ de la calidad de vida ${ }^{16}$. Infortunadamente, los estudios se han conducido de manera casi exclusiva en pacientes con hemodiálisis ${ }^{17,18}$, quienes deben asistir a los centros de salud de forma continua, momento que se aprovecha para la realización de la sesión de ejercicios (acondicionamiento intradiálisis). No ocurre lo mismo con los pacientes con diálisis peritoneal, que sólo asisten a sus respectivos controles médicos, lo cual implica que deben contar con un tiempo adicional para asistir a un programa de acondicionamiento físico y hasta la fecha son pocos los estudios relacionados sobre el efecto del ejercicio físico en la diálisis peritoneal ${ }^{19}$ y la forma en que debe indicarse para este tipo de población ${ }^{17}$. Más aún, a pesar de la dificultad para cumplir con el traslado de los pacientes, se propone una intervención cuya duración es de 18 semanas con frecuencia de tres veces a la semana y con promedio de una hora por sesión.

Con este estudio se intentó determinar el efecto de un programa de acondicionamiento físico sobre los indicadores bioquímicos, la capacidad funcional y la calidad de vida relacionados con la salud en pacientes de diálisis peritoneal. De modo adicional, se emite una propuesta para la prescripción de actividad física sistemática y su desarrollo en pacientes con ERC en diálisis peritoneal.

\section{Materiales y métodos}

Estudio piloto con 11 pacientes desarrollado en un período de 18 semanas (dos semanas de evaluación, una antes y una después) y 16 semanas de intervención, tres sesiones por semana con duración promedio de una hora por sesión.

La población de referencia para este estudio la integraron los pacientes con ERC en diálisis peritoneal de la Clínica Nephrocare El Dorado, de Fresenius Medical Care Colombia, en la ciudad de Bogotá. Los criterios de inclusión para la selección de la población fueron: pacientes mayores de edad ( $\geq 18$ años), que al menos tuvieran tres meses de tratamiento en diálisis peritoneal, afección médica estable avalada por el médico 
tratante, en consenso con el coordinador nacional de diálisis peritoneal. Los criterios de exclusión fueron: hiperglucemia o hipoglucemia no controlada, síntomas cardiovasculares no controlados, compromiso cognitivo que imposibilitara seguir órdenes y amputaciones de miembros inferiores que impidieran el desplazamiento; no se tuvo en cuenta a los pacientes que por diversos motivos no satisficieran la totalidad de las exigencias o se retiraran durante el desarrollo del programa.

Una de las condiciones de participación implicaba el desplazamiento desde su lugar de residencia hasta el centro de salud para la intervención, tres veces por semana, para completar las 48 sesiones.

\section{Principios éticos}

Los pacientes que aceptaron su participación firmaron el consentimiento informado. Los resultados se registraron en la base de datos sin detalles de identificación para mantener la confidencialidad de los participantes. El comité de ética en investigación del Fresenius Medical Care Colombia S.A. avaló la realización de este estudio de acuerdo con los parámetros de la Declaración de Helsinki.

\section{Valoración funcional}

Las pruebas de medición estuvieron interconectadas con:

1. Calidad de vida relacionada con la salud SF-36 $20-22$.

2.Pruebas de capacidad funcional y actividad física: prueba de la marcha de 6 minutos $\left(6 \mathrm{MWT}^{23}\right), 30$ Sit to Stand, dinamometría de mano (dinamómetro digital Takei 5401). Para su evaluación se usó la metodología de la Sociedad Canadiense de Fisiología Deportiva (CSEP 2003), en la que el individuo comprime el dinamómetro con la máxima fuerza posible mediante una contracción máxima breve y sin realizar movimientos corporales adicionales. Se deben intentar tres tomas con cada mano con un minuto de descanso entre cada una y registrar la mejor puntuación del paciente ${ }^{24}$. Se utilizaron la prueba de Sit and Reach y la composición corporal (BCM, Fresenius-Body Composition Measure, Fresenius Medical Care AG and Co. KGaA 61346 Bad Homburg, Germany) $)^{25-27}$.

Para el conteo de pasos diarios se empleó la podometría uniaxial (Omron HJ-002LA Health Care Inc. IL, USA) durante las 16 semanas de intervención.
Los pacientes debían registrar en un diario el número de pasos dados por día. La finalidad de su uso, más allá de seguir o tener en cuenta algún protocolo establecido actualmente, era lograr que tuvieran una mejor adherencia al programa los días que no debían asistir y promover hábitos saludables para ser más activos y por ende reducir el comportamiento sedentario a causa de la ERC.

3. Los indicadores bioquímicos seleccionados en este estudio fueron: nitrógeno ureico sanguíneo (BUN), creatinina, urea, Kt/V, calcio, fósforo, potasio, hemoglobina y hematócrito.

Los resultados de estos parámetros se obtuvieron de la historia clínica de los pacientes seleccionados, como parte de los controles realizados mensualmente de acuerdo con su tratamiento en la Clínica Nephrocare el Dorado.

\section{Programa de acondicionamiento}

El diseño del programa se estructuró en cuatro etapas:

1. Etapa de adaptación (cuatro semanas).

2. Etapa de acondicionamiento (ocho semanas).

3. Etapa de mantenimiento (cuatro semanas).

4. Mediciones (dos semanas, una semana antes y una después de la intervención).

\section{Etapas del programa}

Etapa de adaptación: semanas 1 a 4 (12 sesiones presenciales).

Objetivo: sensibilizar a los pacientes y sus familias a la inclusión e importancia de la actividad física como parte del tratamiento de la ERC. Enseñar la forma adecuada de respirar y la correcta ejecución biomecánica de cada uno de los ejercicios propuestos y las alertas que conlleve suspender la sesión.

\section{EJERCICIOS}

Resistencia cardiovascular: se utilizaron bicicletas estática y recumbente.

Fuerza: ejercicios de cadena cinética cerrada y frenada, flexoextensión de codo, extensión de hombro, press militar, flexión de codo inclinado con apoyo en pared, sentadillas. De la misma forma, durante esta etapa se enfatizó en los ejercicios del CORE ${ }^{28-30}$ (incluidos tórax y abdomen) y las cinturas escapular y pélvica (elevación de cabeza y glúteo). Esto tenía el fin 
de lograr la máxima estabilidad central que les permitiera un mejor desempeño distal.

Etapa de acondicionamiento: semana 5 a 12 (24 sesiones presenciales).

Objetivo: incrementar la resistencia cardiovascular y el desarrollo de la fuerza muscular para alcanzar la realización de actividades funcionales que les permitiera llevar a cabo las actividades de la vida diaria de forma independiente, para mejorar así las variables de calidad de vida. Se incluyó otro ejercicio de equilibrio y coordinación.

\section{EJERCICIOS}

Resistencia cardiovascular: baile con desplazamientos continuos.

Fuerza: abducción horizontal, flexoextensión de hombro, elevación de pierna, caminata tijera, marcha militar.

Etapa de mantenimiento: comprendió las últimas cuatro semanas, 13 a 16 (12 sesiones presenciales).

Objetivo: conservar la condición física adquirida y mantener el tiempo de ejecución hasta lograr la realización de actividades aeróbicas continúas por $30 \mathrm{mi}-$ nutos a través de movimientos con obstáculos similares a los que enfrentan los pacientes en las diferentes actividades de la vida diaria, lo cual implicaba la reducción del grado de fatiga ${ }^{31-34}$.

\section{EJERCICIOS}

Resistencia cardiovascular: baile con desplazamientos continuos; además se realizaron ejercicios con obstáculos. Fuerza: sólo se utilizaron brazos de palanca largos, abducción pierna con flexión de rodilla, extensión de cadera con rodilla flexionada, elevación frontal de pierna, rodilla flexionada y extendida, abducción y aducción de pierna en extensión,

Ejercicios de coordinación visopédica y visomanual: agarre en un solo rebote de fitball, equilibrio sobre en una sola pierna en step, pateo y detención de balón.

\section{Mediciones}

Las pruebas se desarrollaron en dos días y se hicieron de acuerdo con un protocolo en el mismo lugar y hora, durante dos semanas, una antes de iniciar y la otra posterior tras concluir la participación en el programa.
Para conocer más detalles de la forma en que se llevó a cabo el programa de acondicionamiento físico y las mediciones puede consultarse el anexo.

De igual manera, en las sesiones se vigilaron constantemente la frecuencia cardíaca, tensión arterial sistólica y diastólica (TAS-TAD), saturación periférica de oxígeno $\left(\mathrm{SpO}_{2}\right)$, mapeo de pies (evaluación de la sensibilidad superficial a través de los dermatomas en pies) y esfuerzo percibido con la Escala modificada de Borg (escala 10) ${ }^{35-38}$.

De manera adicional, vale la pena aclarar que los horarios en los que se desarrolló el programa de acondicionamiento físico se seleccionaron de común acuerdo con los pacientes, la dirección médica del programa y los investigadores responsables de la intervención: lunes, miércoles y viernes (8 am a $10 \mathrm{am}$ ) y el segundo grupo martes, jueves y sábado (10 am a $12 \mathrm{~m}$ ); esto se efectuó con el fin de que no se afectara el tratamiento durante su participación.

De la misma forma, a lo largo de las 18 semanas que duró la intervención, los pacientes mantuvieron líquido en su cavidad peritoneal y, debido a ello, todas las actividades que se prescribieron se seleccionaron de forma minuciosa. Asimismo, se buscó que fueran funcionales y al mismo tiempo que su efecto fuera mínimo en la salud del paciente, para que de esta manera no hubiera efecto adverso alguno relacionado con el catéter, sino que el ejercicio fuera de su agrado y gusto; todo el tiempo se hallaron bajo la supervisión de los profesionales del ejercicio adscritos al proyecto, los cuales hacían énfasis en la correcta ejecución del movimiento y estaban atentos para que no se extralimitaran (escala de Borg, 0-10), de acuerdo con los objetivos fijados para cada una las etapas.

\section{Análisis estadístico}

Se tuvieron en cuenta sólo los datos de los pacientes que completaron en su totalidad el programa de acondicionamiento físico para realizar un análisis por protocolo.

Se calcularon estadísticas descriptivas con medidas de frecuencia (absoluta y relativa) y medidas de tendencia central. El análisis bivariado por medio de pruebas paramétricas (t de Student para muestras relacionadas) y no paramétricas (prueba de rangos de Wilcoxon). El nivel de significancia estadística se fijó con un valor de $p \leq 0.05$. Los análisis se desarrollaron en el programa SPSS para Windows 22.0 (SPSS Lic. Universidad del Rosario). 


\section{Resultados}

Se incluyó a 11 (100\%) pacientes en diálisis peritoneal desde la clínica Nephrocare El Dorado, de los cuales $5(45.4 \%)$ cumplieron con el $100 \%$ del programa de acondicionamiento físico. Un (9.1\%) individuo se retiró por motivos laborales y los 5 (45.4\%) restantes no cumplieron la totalidad de las sesiones $(\leq 40 \%)$ por causas diversas sin relación con la participación en el programa de acondicionamiento físico, sino con la ERC que padecían y que los había llevado a la diálisis. Las causas por las cuales no completaron la intervención los 6 pacientes (54.6\%) fueron las siguientes: trabajo, retinopatía diabética, anemia no controlada, enfermedad vascular periférica no controlada, problemas familiares y problemas emocionales.

Por tal motivo, la información subsiguiente se basó en la medición y el análisis de los resultados de los 5 pacientes (45.4\%) que cumplieron con la totalidad de las condiciones del estudio: características sociodemográficas, resultados anteriores y posteriores a la prueba de la calidad de vida relacionada con la salud, capacidad funcional y de actividad física, y los indicadores bioquímicos.

\section{Características sociodemográficas}

La edad promedio de la población estudiada fue de $57.8 \pm 3.7$, con la mayoría de pacientes del género masculino, que se hallaban en el régimen de salud contributivo y como modalidad de diálisis peritoneal dominante la DPA, seguida de la DPCA. Las causas vinculadas con la ERC se relacionan con HTA y DM (Tabla 1).

\section{Calidad de vida relacionada con la salud}

Se pudo constatar a través de la aplicación del SF36 , antes y después de la intervención, una tendencia a la mejoría, la cual es estadísticamente significativa en los pacientes que cumplieron en su totalidad la participación en el programa, en particular en los dominios de dolor corporal $(p=0.032)$, rol físico $(p=0.0041)$, función física, vitalidad, salud mental $(p=0.0042)$ y salud general $(p=0.044)$ (Tabla 2).

\section{Capacidad funcional y de actividad física}

Se pudo observar el incremento de forma significativa del número de pasos determinados por el podómetro ( $p=0.050$ ) durante el desarrollo de los 6MWT
Tabla 1. Características demográficas de la población que terminó la intervención

\begin{tabular}{|c|c|}
\hline Variable & Pacientes $(n=5)$ \\
\hline Edad & $57.8 \pm 3.7$ \\
\hline Género Masculino & $3(60 \%)$ \\
\hline Femenino & $2(40 \%)$ \\
\hline $\begin{array}{l}\text { Causa relacionada con la enfermedad renal } \\
\text { crónica (\%) } \\
\text { Hipertensión arterial (HTA) } \\
\text { Diabetes mellitus (DM) } \\
\text { HTA-DM }\end{array}$ & $\begin{array}{l}2(40 \%) \\
2(40 \%) \\
1(20 \%)\end{array}$ \\
\hline $\begin{array}{l}\text { Modalidad de diálisis peritoneal que recibían } \\
\text { DPA } \\
\text { DPCA }\end{array}$ & $\begin{array}{l}3(60 \%) \\
2(40 \%)\end{array}$ \\
\hline $\begin{array}{l}\text { Régimen de salud (\%) } \\
\text { Contributivo } \\
\text { Subsidiado }\end{array}$ & $\begin{array}{l}4(80 \%) \\
1(20 \%)\end{array}$ \\
\hline
\end{tabular}

Variables presentadas como media \pm desviación estándar y porcentaje. Datos obtenidos de los pacientes que cumplieron con la totalidad del programa.

Tabla 2. SF-36 Calidad de vida relacionada con la salud: SF-36 antes y después de la intervención

\begin{tabular}{|c|c|c|c|}
\hline \multicolumn{4}{|c|}{ Calidad de vida relacionada con la salud: SF-36 } \\
\hline Variable & Antes & Después & Valor $\mathbf{p}$ \\
\hline $\begin{array}{l}\text { Variables presentadas } \\
\text { como mediana } \\
\text { Función física } \\
\text { Rol físico } \\
\text { Vitalidad }\end{array}$ & $\begin{array}{l}65 \\
50 \\
60\end{array}$ & $\begin{array}{l}90 \\
50 \\
80\end{array}$ & $\begin{array}{l}0.042^{*} \\
0.041^{*} \\
0.042^{*}\end{array}$ \\
\hline \multicolumn{4}{|c|}{ *Valor estadísticamente significativo } \\
\hline $\begin{array}{l}\text { Variables presentadas } \\
\text { como media } \pm \\
\text { desviación estándar } \\
\text { Dolor corporal } \\
\text { Salud general } \\
\text { Función social } \\
\text { Rol emocional } \\
\text { Salud mental }\end{array}$ & $\begin{array}{c}54.2 \pm 31.8 \\
43 \pm 16.8 \\
60.2 \pm 34.6 \\
44.37 \\
65.33\end{array}$ & $\begin{array}{c}100 \\
80.6 \pm 17.24 \\
100 \\
100 \\
92\end{array}$ & $\begin{array}{c}0.032^{*} \\
0.044^{*} \\
0.062 \\
0.059 \\
0.042^{*}\end{array}$ \\
\hline \multicolumn{4}{|c|}{ *Valor estadísticamente significativo } \\
\hline $\begin{array}{l}\text { Total } \\
\text { SF-36 }\end{array}$ & $54.6 \pm 19.5$ & $93.4 \pm 7.92$ & $0.006^{*}$ \\
\hline *Valor es & ísticamente & nificativo & \\
\hline
\end{tabular}

Datos obtenidos de los pacientes que cumplieron con la totalidad del programa.

(Tabla 3) y el número de veces de sentarse-levantarse en la prueba 30 Sit to Stand $(p=0.004)$ (Tabla 3 ).

Por otro lado, no se presentaron cambios estadísticamente significativos en la prueba de flexibilidad Sit 
Tabla 3. Condición física antes y después de la intervención

\begin{tabular}{|c|c|c|c|}
\hline \multicolumn{4}{|c|}{ Condición física } \\
\hline Variable & Antes & Después & Valor $p$ \\
\hline \multicolumn{4}{|l|}{$\begin{array}{l}\text { Capacidad aeróbica: } \\
\text { marcha de } 6 \text { minutos } \\
\text { (6MWT) }\end{array}$} \\
\hline FC inicial & $82.4 \pm 7.30$ & $72.8 \pm 10.25$ & 0.247 \\
\hline FC final & $108.2 \pm 22.62$ & $91.2 \pm 12.31$ & 0.212 \\
\hline $\begin{array}{l}\text { Distancia } \\
\text { recorrida (m) }\end{array}$ & 475 & 488 & 0.08 \\
\hline TAS inicial $(\mathrm{mm} / \mathrm{Hg})$ & $146.6 \pm 26.27$ & $151.4 \pm 29.72$ & 0.816 \\
\hline TAD inicial $(\mathrm{mm} / \mathrm{Hg})$ & $86.6 \pm 10.85$ & $89.6 \pm 11.43$ & 0.746 \\
\hline TAS final $(\mathrm{mm} / \mathrm{Hg})$ & $164.8 \pm 3.5$ & $173.4 \pm 42.88$ & 0.415 \\
\hline TAD final $(\mathrm{mm} / \mathrm{Hg})$ & $87^{\wedge}$ & 82 & 0.5 \\
\hline $\begin{array}{l}\text { Número de pasos } \\
\text { durante la prueba } \\
\text { (podómetro) }\end{array}$ & $546.8 \pm 216.1$ & $860.8 \pm 329.43$ & $0.050^{*}$ \\
\hline \multicolumn{4}{|l|}{$\begin{array}{l}\text { Fuerza prensil: } \\
\text { dinamometría de } \\
\text { mano (handgrip) }\end{array}$} \\
\hline $\begin{array}{l}\text { Mejor intento mano } \\
\text { izquierda }(\mathrm{kg})\end{array}$ & $17.68 \pm 3.5$ & $20.66 \pm 4.26$ & $\begin{array}{c}0.371 \\
0\end{array}$ \\
\hline $\begin{array}{l}\text { Mejor intento mano } \\
\text { derecha }(\mathrm{kg})\end{array}$ & $18.78 \pm 5.07$ & $19.68 \pm 6.54$ & .347 \\
\hline $\begin{array}{l}30 \text { Sit to Stand } \\
\text { Repeticiones (\#) }\end{array}$ & $9 \pm 3.74$ & $21.6 \pm 3.84$ & $0.004^{*}$ \\
\hline $\begin{array}{l}\text { Flexibilidad: Sit and } \\
\text { Reach }\end{array}$ & & & \\
\hline & $7 \pm 9.61$ & $5.20 \pm 5.76$ & 0.455 \\
\hline
\end{tabular}

FC: frecuencia cardíaca; TAS: tensión arterial sistólica; TAD: tensión arterial diastólica; Spo2: oximetría de pulso.

Variables presentadas como media \pm desviación estándar y porcentaje. ^Mediana. *Valor estadísticamente significativo.

Datos obtenidos de los pacientes que cumplieron con la totalidad del programa.

and Reach ni en la prueba de dinamometría de mano Handgrip (Tablas 3 y 4).

El resumen de la medición anterior y posterior a la prueba se muestra en las Tablas 3 y 4.

De la misma forma, el promedio de pasos realizados por los pacientes durante el estudio (123 días) fue de 5,171 diarios, lo cual sugiere un aumento de forma paulatina de la actividad física realizada durante la intervención por los pacientes que completaron el estudio.

Si se considera el control de los signos vitales realizado a los pacientes durante las 48 sesiones, se registraron la estabilización y la disminución de la FC inicial, junto con las presiones sistólica y diastólica (Tabla 5).

\section{Indicadores bioquímicos}

No se identificaron cambios estadísticamente significativos en los parámetros bioquímicos (Tabla 6) después de las 16 semanas de intervención. No se
Tabla 4. Composición corporal antes y después de la intervención

\begin{tabular}{|l|c|c|c|}
\hline \multicolumn{4}{|c|}{ Composición corporal } \\
\hline Variable & Antes & Después & Valor p \\
\hline $\begin{array}{l}\text { Peso (kg) } \\
53.7 \pm 8.23\end{array}$ & $53.74 \pm 8.23$ & 0.985 \\
\hline $\begin{array}{l}\text { Índice de masa } \\
\text { corporal (IMC) }\end{array}$ & $16.77 \pm 2.36$ & $16.78 \pm 2.37$ & 0.978 \\
\hline $\begin{array}{l}\text { Porcentaje } \\
\text { graso (\%) }\end{array}$ & $29.5 \pm 9.31$ & $30 \pm 8.93$ & 0.713 \\
\hline $\begin{array}{l}\text { Porcentaje magro (\%) } \\
\text { Sobrehidratación (oh) }\end{array}$ & $55.3 \pm 9.91$ & $55.82 \pm 10$ & 0.743 \\
\hline
\end{tabular}

Variables presentadas como media \pm desviación estándar.

Datos obtenidos de los pacientes que cumplieron con la totalidad del programa.

Tabla 5. Resumen del control de signos vitales durante las 48 sesiones

\begin{tabular}{|l|c|c|}
\hline Variable & Media & DS \\
\hline FC inicial & 91 & 21.7 \\
\hline FC máx & 93 & 33.2 \\
\hline FC post & 81 & 24 \\
\hline TAS inicial & 135 & 44 \\
\hline TAD inicial & 80 & 23.3 \\
\hline TAS máx & 127 & 43.2 \\
\hline TAD máx & 76 & 25.4 \\
\hline TAS post & 125 & 38.2 \\
\hline TAD post & 77 & 22.7 \\
\hline Spo ${ }_{2}$ inicial & 87 & 23.7 \\
\hline Spo $_{2}$ máx & 85 & 26 \\
\hline
\end{tabular}

FC: frecuencia cardíaca; TAS: tensión arterial sistólica; TAD: tensión arterial diastólica; $\mathrm{SpO}_{2}$ : oximetría de pulso.

Variables presentadas como media \pm desviación estándar.

Datos obtenidos de los pacientes que cumplieron con la totalidad del programa.

presentaron efectos adversos en los pacientes que participaron en la intervención, relacionados o cuya razón principal fuera su participación en el programa de acondicionamiento físico.

Por otro lado, luego de comparar los registros de antecedentes de salud antes y después de la intervención, y junto con el concepto del médico tratante, es importante comentar que al $40 \%$ de los pacientes (2) que finalizaron el programa se le suspendió el consumo del hierro sacarato y la eritropoyetina, y su mejoría se relacionó con el incremento de la actividad física y la participación en este programa. 
Tabla 6. Indicadores bioquímicos antes y después de la intervención

\begin{tabular}{|l|c|c|c|}
\hline \multicolumn{4}{|c|}{ Indicadores bioquímicos } \\
\hline Variable & Antes & Después & Valor p \\
\hline BUN (mg/dl) & $54.6 \pm 10.4$ & $60.96 \pm 19.39$ & 0.556 \\
\hline (Hb) (g/dl) & $12 \pm 2.48$ & $9.9 \pm 6.12$ & 0.417 \\
\hline Kt/V & $2.49 \pm 0.51$ & $2.17 \pm 0.27$ & 0.102 \\
\hline Urea (mg/dl) & $116 \pm 22.9$ & $130.4 \pm 41.49$ & 0.528 \\
\hline Calcio (mg/dl) & $9.94 \pm 1.23$ & $9.72 \pm 0.85$ & 0.462 \\
\hline Fósforo (mg/dl) & $4.75 \pm 0.48$ & $5.11 \pm 1.12$ & 0.562 \\
\hline Potasio (mmol/dl) & $4.87 \pm 1.09$ & $4.54 \pm 0.85$ & 0.166 \\
\hline Hematócrito (\%) & $36.84 \pm 6.77$ & $37.7 \pm 7.35$ & 0.481 \\
\hline
\end{tabular}

BUN: nitrógeno ureico; $\mathrm{Hb}$ : hemoglobina.

Variables presentadas como media \pm desviación estándar y porcentaje.

Datos obtenidos de los pacientes que cumplieron con la totalidad del programa.

\section{Discusión}

Este estudio piloto evaluó el efecto de un programa de acondicionamiento físico sobre los indicadores bioquímicos, la capacidad funcional y la calidad de vida relacionada con la salud de un grupo de pacientes con DP. Los hallazgos más importantes se presentaron respecto de la calidad de vida relacionada con la salud, a través de la mejoría en las puntuaciones de las dimensiones: dolor corporal, rol físico, función física, vitalidad, salud mental y salud general, medidas con el SF-36, y claramente muestran que luego de completar una intervención de un programa de ejercicio físico se identifica un cambio favorable, no sólo en la percepción física, social y psicológica sino también en el estado de salud general de los pacientes que completaron la intervención, lo que contribuye a una mejor sensación de bienestar en éstos.

En cuanto a la capacidad funcional y actividad física, se evidenció un incremento del número de pasos dados durante la 6MWT y en los pasos diarios (5,171 en promedio) dados por los pacientes durante toda la intervención; esto supera el resultado del estudio de Daisuke del 2012, en el cual los pacientes lograron en promedio 4,367 pasos. Asimismo, se incrementó el número de veces de sentarse-levantarse, lo cual se advirtió cuando los participantes retomaron por sí mismos las actividades y funciones de la vida diaria que desempeñaban con anterioridad y que dejaron de realizar a causa de la ERC.
De igual forma, es relevante comentar que luego de comparar los registros de antecedentes de salud antes y después de la intervención, junto con el concepto del nefrólogo tratante, a $2(40 \%)$ de los pacientes que completaron el programa se le suspendió el consumo del hierro sacarato y la eritropoyetina, en relación con su mejoría con el incremento de la actividad física y su participación en este programa. Hasta el momento no se ha notificado lo anterior en los estudios similares efectuados en pacientes con DP.

Además, se logró la estabilización y disminución de la FC inicial, junto con las presiones sistólica y diastólica, lo cual puede significar una mejor y mayor adherencia al tratamiento. Estos resultados obtenidos son importantes para conducir estudios futuros acerca de los programas de ejercicio físico sistemático y su incidencia en la estabilidad del tratamiento, como su función protectora.

Al mismo tiempo, la propuesta de este estudio para la prescripción de ejercicio físico sistemático, y su desarrollo exclusivo en pacientes con DP, revela la escasez de estudios relacionados con la institución de programas de este tipo para esta población. De acuerdo con la revisión efectuada en diferentes bases, entre los años 2000 y 2018 se registraron sólo 10 estudios $^{39-48}$.

En realidad, sólo Mustata, et al. aplicaron en el año 2005 un programa de Tai chi Chuan estilo Wu (un tipo de ejercicio de leve a moderado), con la intención de promover el equilibrio en lugar de la fuerza, y la concentración en vez de la velocidad, a través de clases semanales de 1 hora de duración durante tres meses dirigidas en la unidad renal. Straub, en el 2008, propuso un programa de acondicionamiento físico en casa sin control directo de ocho semanas de duración, consistente en actividades como nadar, montar bicicleta y caminar.

Según Bennett, et al. en el 2017, esto se debe a una serie de factores que dificultan y al mismo tiempo impiden que el ejercicio físico pueda incluirse como parte del tratamiento que reciben las personas con ERC que están en TRR ${ }^{49}$; esto se relaciona, por un lado, con la falta de familiarización con el tema y sus beneficios en esta población de los profesionales de la salud que los atienden, los propios pacientes, sus familiares, los cuidadores, la comunidad académica, los tomadores de decisiones y el sistema general de salud. Por otro lado, se encontró la misma condición física de los pacientes afectados por la ERC que padecen y como consecuencia el sedentarismo que propicia ésta, lo que lleva a la desmotivación, fatiga y cansancio durante las primeras sesiones y que produce una complejidad en el 
desarrollo y el manejo de ellos en los estudios, tal y como demostraron O' Coonor, et al. en el 2017.

Debido a ello, la muestra de pacientes participantes tiende a ser pequeña, como en este estudio y también en los protocolos de Straub, et al. del 2008 con cinco pacientes; Mustata, et al. del 2005 con nueve pacientes; Oishi, et al. del 2012 con 38 pacientes; y Brodin, et al. del 2001 con 33 pacientes. Además, señalan que son pocos los pacientes que pueden cumplir con los requerimientos del estudio.

También se advirtió que, debido al comportamiento progresivo y agresivo de la ERC, propicia una escasa adherencia por parte de los pacientes participantes en las intervenciones, lo que lleva a no completar y al retiro de éstas ${ }^{50}$. Así lo confirman también Straub, et al. en el 2008 y Mustata, et al. en el 2005 (tres participantes retirados). Es importante destacar que dentro de las causales no hubo efectos adversos secundarios al acondicionamiento físico. Esto muestra una vez más que el ejercicio físico sistemático, prescrito y dirigido por profesionales cualificados no representa contratiempo alguno.

En cuanto a la condición física de los pacientes con DP, se han utilizado diversas pruebas para determinar cuál cuantifica de forma objetiva las variables: funcionamiento físico, capacidad funcional, efecto de la ERC en éstas y las modificaciones que presentan luego de un programa de ejercicio físico, como Painter ${ }^{51}$, Straub $^{40}$ y Oishi ${ }^{44}$, Scatolin ${ }^{39}$, Breiterman-White ${ }^{52}$, Bro$\operatorname{din}^{41}$, Ming-Liang ${ }^{47}$ y Enia ${ }^{43}$.

Las pruebas que se han utilizado en estos estudios son el Short Physical Performance Battery (SPPB) ${ }^{40}$, cicloergometría ${ }^{42}$, espirometría ${ }^{42}$, prueba de la marcha de 30 metros $^{41}$, dinamometría de mano ${ }^{41}$ y la podometría ${ }^{44}$. Pero éstas a su vez han sido limitadas e insuficientes, ya que desafortunadamente no se han validado para emplearse en esta población, con el fin de determinar los baremos que optimicen el análisis, el seguimiento y el control.

Por otro lado, en cuanto a los parámetros bioquímicos, éste y otros estudios coinciden en utilizarlos como medio de control para observar el efecto en los individuos que se encuentran con DP posterior a su participación en un programa de hábitos saludables (Brodin et al, en 2000), acondicionamiento físico (Straub, et al. en 2008; Shahgholian, et al, en 2015; Daisuke, et al. en el 2012), el mismo efecto negativo generado por la ERC que padecen (Ulubay en el 2006; Enia, et al. en el 2012), o relacionan la habilidad funcional y la tolerancia con el acondicionamiento y la presencia de anemia $^{52,53}$. Este estudio concuerda con el de
Mustata, et al. del 2005 al medir hemoglobina, potasio, calcio, fósforo, creatinina y relacionar la dosis de diálisis adecuada y su nexo con la condición física y la calidad de vida vinculada con la salud, en coherencia con la eficacia de su tratamiento.

No se observaron cambios en este estudio en los indicadores bioquímicos, pero se reconoció un aumento de los valores de urea y fósforo, junto con una disminución de la hemoglobina en los pacientes que concluyeron su participación, lo cual se vincula con la conducta de la ERC ${ }^{54-56}$, como lo mostraron Kalantar, et al. en el 2003, Pendse, et al. en el 2005, Torregrosa, et al. en el 2011 y Hruskay, et al. en el 2017. Por otra parte, en cuanto a la calidad de vida relacionada con la salud de los pacientes, sólo tres estudios de Brodin, et al. del 2000 y Mustata, et al. del 2005, y éste mismo, evaluaron dicho aspecto a través del SF- $36^{48}$ y el Nottingham Health Profile (NHP) ${ }^{41}$. Dentro de los hallazgos de este estudio mediante el SF-36 se encuentra una mejoría significativa en las dimensiones de dolor corporal, rol físico, función física, vitalidad, salud mental, salud general y puntuación total de SF-36, mientras que los resultados más relevantes que obtuvieron Mustata, et al. en el 2005 en su intervención fueron función social y rol emocional, los cuales son los más afectados en esta población, tal y como lo muestra el estudio de Brodin, et al. en el 2000, en el que se usó el NHP ${ }^{41}$.

Si bien en la bibliografía actual se encuentran múltiples estudios relacionados con la calidad de vida de este tipo de población, se han conducido con muestras pequeñas; en consecuencia, deben impulsarse nuevos estudios con un número significativo de pacientes que analicen la relación que existe entre los indicadores bioquímicos de condición física y el efecto sobre la calidad de vida, para aportar evidencia relacionada con el manejo estandarizado de esta población.

Por último, para que haya una mejor adherencia de los pacientes a este tipo de intervenciones, deben acompañarse de una serie de procesos, los cuales aseguran que su participación sea más exitosa y agradable y se logre al mismo tiempo su cumplimiento; se describen a continuación.

Educativos. Se les explica a todas las personas la importancia del ejercicio físico en la ERC, los beneficios de su práctica regular, la relevancia de incluirlo como parte de sus estilos de vida y, de igual manera, en qué consiste el programa de acondicionamiento físico que se desarrollará con los pacientes.

Motivacionales. Es importante que los pacientes se sientan partícipes en la intervención y por tal motivo es posible fijar en su desarrollo una serie de 
reconocimientos morales, con énfasis en su participación y los objetivos propuestos alcanzados, como el resultado de las valoraciones periódicas; esto acentúa el grado de compromiso y participación en el programa.

Empoderamiento. En los días que los pacientes no asistan al centro de diálisis, deben realizar actividades complementarias como parte de la intervención; esto contribuye a crear un estilo activo y saludable y sirve además para conocer su grado de compromiso, tal y como lo hicieron Mustata, et al. en 2005 con las secuencias de tai chi que realizaban en sus hogares y, en este estudio, con el podómetro. Es por ello importante la participación activa de los familiares y cuidadores para que sea exitosa.

Acompañamiento. Durante la intervención siempre es necesaria la presencia de un profesional del ejercicio físico que resuelva todas las dudas y de igual manera dirija las actividades a realizar en el programa.

Evaluación. Es importante conocer la evolución que consigan los participantes en el programa y por tanto las pruebas deben realizarse de forma periódica. Asimismo, deben considerarse las sugerencias, comentarios y aspectos por mejorar realizados en el programa para su fortalecimiento continuo.

\section{Limitaciones}

Las principales limitaciones de este estudio son el pequeño tamaño de la muestra, la realización de un muestreo no probabilístico y la falta de disponibilidad de un grupo control, que no permite establecer comparaciones dentro del mismo estudio.

Desde el punto de vista de la logística, una de las limitaciones fue la dificultad para el desplazamiento de los pacientes que requerían un acompañante en las instalaciones donde se desarrolló el programa, en virtud del costo adicional en que se debía incurrir.

\section{Conclusión}

Este estudio piloto ha demostrado que 18 semanas de un programa de acondicionamiento físico, que incluya además actividades de resistencia cardiovascular y fuerza complementadas con procesos educativos contribuyen al mejoramiento significativo de los siguientes dominios en la calidad de vida vinculada con la salud evaluados a través del SF-36: dolor corporal, rol físico, función física, vitalidad, salud mental y salud general, así como la capacidad funcional en este tipo de población.
Lo anterior se reflejó en particular cuando los pacientes realizaban diferentes actividades de la vida diaria por sí mismos sin ayuda de otras personas, como también al retomar diferentes roles en los que interactuaban y se desempeñaban antes de la ERC.

Los cambios significativos en la calidad de vida relacionada con la salud se evidenciaron en una mayor y mejor adherencia a la TRR y confirmaron los postulados de diferentes investigadores que proponen la inclusión del acondicionamiento físico como parte del tratamiento de los pacientes con ERC y DP; esto lo convierte en un factor protector de la salud y modifica los indicadores de salud pública.

Asimismo, es innegable considerar que el acondicionamiento físico debe incluirse en las guías nacionales e internacionales para el tratamiento de los pacientes con ERC.

Por último, es necesario realizar otros estudios que contribuyan no sólo a generar el conocimiento sino la evidencia necesaria para prescribir ejercicio físico al desarrollar programas de acondicionamiento físico, como parte del tratamiento que reciben los pacientes con ERC; esto debe acompañarse de la validación de pruebas y baterías con sus respectivos baremos, que se adapten y puedan utilizarse para evaluar y medir objetivamente este tipo de población.

\section{Agradecimientos}

Los autores agradecen especialmente a los doctores Jesús Muñoz y Alonso Gómez, de Fresenius Medical Care Colombia, y a la doctora Juanita Castro, de la Fundación Fresenius, por su incondicional apoyo e invaluable contribución y colaboración; de igual manera, extienden su agradecimiento a los estudiantes de la Facultad de Cultura Física, Deporte y Recreación de la Universidad Santo Tomás del programa de pregrado y la Maestría en Actividad Física para la Salud, sin dejar de lado al personal médico, asistencial, administrativo y a los pacientes de diálisis peritoneal de la Clínica Nephro Care el Dorado en Bogotá de Fresenius Medical Care Colombia, ya que sin su participación no habría sido posible este estudio.

\section{Conflicto de intereses}

El Dr. Tobón es empleado de Fresenius Medical Care, pero no se afecta el criterio en la elaboración del artículo; el resto de autores declara no tener conflicto de intereses. 


\section{Financiamiento}

El proyecto recibió financiamiento de la Fundación

Fresenius, pero el aporte económico no influyó en la elaboración del manuscrito.

\section{Responsabilidades éticas}

Protección de personas y animales. Los autores declaran que los procedimientos seguidos se conformaron a las normas éticas del comité de experimentación humana responsable y de acuerdo con la Asociación Médica Mundial y la Declaración de Helsinki.

Confidencialidad de los datos. Los autores declaran que han seguido los protocolos de su centro de trabajo sobre la publicación de datos de pacientes.

Derecho a la privacidad y consentimiento informado. Los autores han obtenido el consentimiento informado de los pacientes o sujetos referidos en el artículo. Este documento obra en poder del autor de correspondencia.

\section{Bibliografía}

1. Bello AK, Levin A, Tonelli M, Okpechi IG, Feehally J, Harris D, et al Global kidney health atlas: a report by the International Society of Nephrology on the current state of organization and structures for kidney care across the globe. International Society of Nephrology, Brussels, Belgium. 2017.

2. Li P, Chow K, Van De Luijtgaarden M, Jager K, Johnson D, Mehrotra R, et al. Changes in the worldwide epidemiology of peritoneal dialysis. $\mathrm{Na}$ Rev Nephrol. 2017;13(2):90-103. doi: 10.1038/nrneph.2016.181.

3. Cuenta de alto costo. Situación de la enfermedad renal crónica, hipertensión arterial y diabetes mellitus en Colombia 2017. Fondo Colombiano de Enfermedades de Alto Costo, 2018. Disponible en: https://cuentadealtocosto.org/site/images/Publicaciones/Situacion_ERC HA_DM_Colombia_2017.pdf

4. Bello AK, Alrukhaimi M, Ashuntantang GE. Complications of CKD: current state, knowledge gaps, and strategy for action. Kidney Int Suppl. 2017;7:122-129. doi:10.1016/i.kisu.2017.07.007.

5. Kalantar-Zadeh K, Stenvinkel P, Pillon L, Kopple J. Inflammation and nutrition in renal insufficiency. Adv Ren Replace Ther. 2003;10(3):155169. doi:10.1053/j.arrt.2003.08.008.

6. Walker SR, Tangri N, Wagner M. Chronic kidney disease, frailty, and unsuccessful aging: a review. J Ren Nutr. 2014;24(6):364-370. https:// doi-org.ez.urosario.edu.co/10.1053/j.jrn.2014.09.001.

7. Campos AY. Ejercicio terapéutico factor protector en pacientes con enfermedad renal crónica. Entérese, Ed: Universidad Manuela Beltrán. 2007; v.23 fasc.1, p.7-22.

8. Manfredini F, Mallamaci F, Catizone L, Zoccali C. The burden of physical inactivity in chronic kidney disease: is there an exit strategy? Nephro Dial Transplant. 2012;27(6):2143-2145. https://doi/10.1093/ndt/gfs120.

9. Eknoyan G, Lameire N, Barsoum R, Eckardt K, Levin A, Levin N, et al. The burden of kidney disease: improving global outcomes. Kidney Int. 2004;66(4):1310-1314. doi: 10.1111/j.1523-1755.2004.00894.x.

10. Institute for Health Metrics and Evaluation (IHME). Rethinking development and health: findings from the Global Burden of Disease Study. Seattle, WA: IHME, 2016

11. Poppe C, Hanoulle I, Vogelaers D, Crombez G, Petrovic M. Improving quality of life in patients with chronic kidney disease: influence of acceptance and personality. Nephrol Dial Transplant. 2013;28(1):116-121. doi: 10.1093/ndt/gfs151.

12. Kutner N. Promoting functioning and well-being in older CKD patients: review of recent evidence. Int Urol Nephrol. 2008;40(4):1151-1158. doi: 10.1007/s11255-008-9469-x.

13. Cheema B, Singh M. Exercise training in patients receiving maintenance hemodialysis: a systematic review of clinical trials. Am J Nephrol. 2005;25(4):352-364. dOI: 10.1159/000087184.
14. Heiwe $\mathrm{S}$, Jacobson $\mathrm{SH}$. Exercise training for adults with chronic kidney disease. Cochrane Database Syst Rev. 2011;Issue 10. Art. No.: CD003236. doi: 10.1002/14651858.CD003236.pub2.

15. Koufaki P, Greenwood SA, Macdougall I C, Mercer TH. Exercise therapy in individuals with chronic kidney disease: a systematic review and synthesis of the research evidence. Annu Rev Nurs Res. 2013;31:235-275. doi:10.1891/0739-6686.31.235.

16. Bennett PN, Breugelmans L, Barnard R, Agius M, Chan D, Fraser D, et al. Sustaining a hemodialysis exercise program: a review. Semin Dial. 2010;vol. 23(1):62-73. doi: 10.1111/j.1525-139X.2009.00652.x.

17. Birinder SC, Danwin C. Resistance training in chronic renal failure In: Ciccolo J, Kraemer W. Resistance training for the prevention and treatment of chronic disease. 2013;77-98. United States: CRC Press.

18. Mercer $T$. Exercise training for individuals with advanced chronic kidney disease. In: Kopple J, Massry S, Kalantar K (Academic Press). Nutritional Management of Renal Disease. 2013:739-773. United States: Elsevier. https://doi.org/10.1016/B978-0-12-391934-2.00045-X.

19. Thangarasa T, Imtiaz R, Hiremath S, Zimmerman D. Physical activity in patients treated with peritoneal dialysis: a systematic review and meta-analysis. Can J Kidney Health Dis. 2018;5:2054358118779821. https:// doi10.1177/2054358118779821.

20. Ricardo AC, Hacker E, Lora CM, Ackerson L, DeSalvo KB, et al. Validation of the kidney disease Quality of Life Short Form 36 (KDQOL-36TM) US spanish and english versions in a Cohort of hispanics with chronic kidney disease. Ethn Dis. 2013.23(2):202-209.

21. Lugo LH, García HI, Vera CY. Confiabilidad del cuestionario de calidad de vida en salud SF-36 en Medellín, Colombia/Reliability of sf-36 quality of life in health questionnaire in Medellín, Colombia. Rev Fac Nac Salud Pública 2006;24(2):37-50. Available from: <http://www.scielo.org.co/scielo.php?script=sci_arttext and pid=S0120-386X2006000200005 and In$\mathrm{g}=\mathrm{en}$ and $\mathrm{nrm}=\mathrm{iso}>$. ISSN 0120-386X.

22. Chaves $\mathrm{K}$, Duarte A, Vesga J. Adaptación transcultural del cuestionario KDQOL SF 36 para evaluar calidad de vida en pacientes con enfermedad renal crónica en Colombia. Revista Med. 2013;21(2):12-17. https://doi. org/10.18359/rmed.1170.

23. Shi Y, Zheng D, Zhang L, Yu Z, Yan H, Ni Z, et al. Six-minute walk test predicts all-cause mortality and technique failure in ambulatory peritoneal dialysis patients. Nephrology (Carlton). 2017;22(2):118-24. doi: 10.1111/ nep.12726.

24. Canadian Society for Exercise Phyisology. The canadian physical activity fitness and lifestyle approach: CSEP-Health and Fitness Program's Health-Realted Appraisal and Conselling Strategy. $3^{\text {rd }}$ ed. Otawa, ON, 2003

25. Wabel $P$, Chamney $P$, Moissl $U$, Jirka $T$. Importance of whole-body bioimpedance spectroscopy for the management of fluid balance. Blood Purif. 2009; 27(1):75-80. doi: 10.1159/000167013.

26. Devolder I, Verleysen A, Vijt D, Vanholder R, Van Biesen W. Body composition, hydration, and related parameters in hemodialysis versus peritoneal dialysis patients. Perit Dial Int. 2010;30(2):208-214.doi:10.3747/ pdi.2008.00284.

27. MoissI UM, Wabel P, Chamney PW. Body fluid volume determination via body composition spectroscopy in health and disease. Physiol Meas. 2006; 27(9):921-933, doi:10.1088/0967-3334/27/9/012.

28. Akuthota V, Nadler SF. Core strengthening. Arch Phys Med Rehabil. 2004; 85(3 Suppl 1): S86-92. doi:10.1053/j.apmr.2003.12.005.

29. Akuthota V, Ferreiro A, Moore $T$, Fredericson M. Core stability exercise principles. Curr Sports Med Rep. 2008;7(1):39-44. doi: 10.1097/01. CSMR.0000308663.13278.69

30. Granacher U, Muehlbauer T, Gollhofer A, Hortobágyi T, Kressig R. The importance of trunk muscle strength for balance, functional performance, and fall prevention in seniors: a systematic review. Sports Med. 2013;43(7), 627-641. doi: 10.1007/s40279-013-0041-1.

31. Jhamb M, Weisbord S, Steel, J, Unruh, M. Fatigue in patients receiving maintenance dialysis: a review of definitions, measures, and contributing factors. Am J Kidney Dis. 2008;52(2):353-365. doi:10.1053/j. ajkd.2008.05.005

32. Bossola M, Vulpio C, Tazza L. Fatigue in chronic dialysis patients. Semin Dial. 2011;24(5):550-555. doi: 10.1111/j.1525-139X.2011.00956.x.

33. Artom M, Moss-Morris R, Caskey F, Chilcot J. Fatigue in advanced kidney disease. Kidney Int. 2014;86(3):497-505. doi: 10.1038/ki.2014.86.

34. Picariello F, Moss-Morris R, Macdougall I. C, Chilcot J. The role of psychological factors in fatigue among end-stage kidney disease patients: a critical review. Clin Kidney J. 2017;10(1):79-88. doi:10.1093/ckj/sfw113.

35. Michael J Chen, Xitao Fan, Sondra T Moe. Criterion-related validity of the Borg ratings of perceived exertion scale in healthy individuals: a meta-analysis. J Sports Sci. 2002;20:11:873-899, doi: $10.1080 / 026404102320761787$.

36. Faulkner J, Eston RG. Perceived exertion research in the $21^{\text {st }}$ century: developments, reflections and questions for the future. J Exerc Sci Fitness. 2008:6(1):1-14.

37. Morishita S, Yamauchi S, Fujisawa C, Domen K. Rating of perceived exertion for quantification of the intensity of resistance exercise. Int $\mathrm{J}$ Phys Med Rehabil. 2013;1:172. doi:10.4172/2329-9096.1000172. 
38. Pereira G, Souza D, Reichert F, Smirmaul B. Evolution of perceived exertion concepts and mechanisms: a literature review. Rev Bras Cineantropom Desempenho Hum. 2014;16(5):579-587. Available from: http:// www.scielo.br/scielo.php?script=sci_arttext and $\mathrm{d}=\mathrm{S} 1980 \quad 00372014000500579$ and Ing=en. doi:10.5007/1980$0037.2014 \mathrm{v} 16 \mathrm{n} 5 \mathrm{p} 579$.

39. Scatolin B, de Vechi A, Ribeiro D, Bertolin D, Canova J, Cesario C, de Cássia R. Atividade de vida diária dos pacientes em tratamento de diálise peritoneal intermitente com cicladora. Arq Ciênc Saúde.2010 janmar; 17(1):15-21. Available from: http://www.ijdr.in/article.asp?iss$\mathrm{n}=09709290$; year $=2008$; volume $=19$; issue $=4$; page $=288$; epage $=291$; aulast=Sanjay.

40. Straub CK, Murphy SO, Rosenblaum R. Exercise in the management of fatigue in patients on peritoneal dialysis. Nephrol Nurs J. 2008;35(5):469-475

41. Brodin E, Ljungman S, Hedberg M, Sunnerhagen KS. Physical activity, muscle performance and quality of life in patients treated with chronic peritoneal dialysis. Scan J Urol Neohrol. 2001;35:71-78. doi:10.1080/00365590151030886.

42. Ulubay G, Akman B, Sezer S, Calik K, Oner F, Ozdemir N, Haberal M Factors affecting exercise capacity in renal transplantation candidates on continuous ambulatory peritoneal dialysis therapy. Transplant Proc. 2006;38(2):401-405. doi:10.1016/j.transproceed.2005.12.107.

43. Enia G, Tripepi R, Panuccio V, Torino C, Garozzo M, Battaglia GG Zoccali C. Pulmonary congestion and physical functioning in peritoneal dialysis patients. Perit Dial Int. 2012;32(5):531-536. doi: 10.3747/ pdi.2010.00250

44. Daisuke O, Koitabashi K, Hikary K, Imai N, Sakurada T, Konno Y, et al. Physical activity is associated with serum albumin in peritoneal dialysis patients. Adv Perit Dial.2012;28:148-152.

45. Krishna SR, Madhavi K, Kishore CK, Kumar VS. Effect of exercise program on lumbosacral strain in patients on continuous ambulatory peritoneal dialysisIndian. J Nephrol. 2014;24(4):262-263. doi:10.4103/09714065.133787.
46. Shahgholian N, KarimiFard O, Shahidi S. Effects of aerobic exercise on blood glucose in continuous ambulatory peritoneal dialysis patients. Iran J Nurs Midwifery Res. 2015;20(2):165-170.

47. Zuo M, Yue WS, Yip T. Prevalence of and associations with reduced exercise capacity in peritoneal dialysis patients. Am J Kidney Dis. 2013;62 (5):939-946. doi 10.1053/j.ajkd.2013.05.016

48. Mustata S, Simon N, Jassal S, Oreopoulos D, Cooper L, Langrick N. The effect of a Tai Chi exercise program on quality of life in patients on peritoneal dialysis: a pilot study. Perit Dial Int. 2005;25(3):291-294.

49. Bennett $P$, Capdarest-Arest N, Parker K. The physical deterioration of dialysis patients-Ignored, ill-reported, and ill-treated. Semin Dial. 2017;Sep;30(5):409-412. doi:10.1111/sdi.12610.

50. O' Coonor E, Macdougall I, Greenwood S. Behavioural change and exercise therapy for patients with CKD. Journal of Kidney Care. 2017;2(2):58-65. https://doi.org/10.12968/jokc.2017.2.2.58.

51. Painter $P$, Marcus R. Physical function and gait speed in patients with chronic kidney disease. Nephrol Nurs J. 2013;40(6):529-538.

52. Breiterman-White R. Functional ability of patients on dialysis: the critical role of anemia. Nephrol Nurs J. 2005;32(1):79-82.

53. Mancini DM, Kunavarapu C. Effect of erythropoietin on exercise capacity in anemic patients with advanced heart failure: management of comorbidities in kidney disease in the $21^{\text {st }}$ century: anemia and bonne disease. Kidney Int Suppl. 2003;64:S48-S52. doi:10.1046/j.1523-1755.64s87.8.x.

54. Pendse S, Singh AK. Complications of chronic kidney disease: anemia, mineral metabolism, and cardiovascular disease. Med Clin North Am. 2005;89(3):549-561. doi: 10.1016/j.mcna.2004.12.004.

55. Hruska KA, Sugatani T, Agapova O, Fang Y. The chronic kidney disease-mineral bone disorder (CKD-MBD): advances in pathophysiology. Bone. 2017; 100:80-86. doi: 10.1016/j.bone.2017.01.023.

56. Torregrosa J, Bover J, Andía J. Recomendaciones de la Sociedad Española de Nefrología para el manejo de las alteraciones del metabolismo óseo-mineral en los pacientes con enfermedad renal crónica (SEN-MM). Nefrología. 2011;31(Suppl.1):3-32. doi:10.3265/Nefrologia.pre2011.Jan.10816. 


\section{Anexo 1 \\ Programa de acondicionamiento físico en pacientes de diálisis peritoneal: un estudio piloto}

\section{Etapas del Programa}

Etapa de adaptación: (Semana $1-4),(12$ sesiones presenciales).

Meta: sensibilizar a los pacientes y sus familias hacia la inclusión e importancia de la actividad física como parte del tratamiento de la ERC. Enseñar la forma adecuada de respirar y la correcta ejecución biomecánica de cada uno de los ejercicios propuestos y las alertas que conlleven suspender la sesión.

Durante la primera semana, se realizaron los ajustes para asignar la carga adecuada individual de cada uno de los ejercicios de fuerza y la selección de los equipos que eran del agrado para cada participante.

\section{Modo}

Resistencia cardiovascular: se trabajó con bicicleta estática y bicicleta recumbent.

Fuerza: mancuernas de 1, 2, 3,4, 6 libras.

\section{Intensidad}

Frecuencia cardiaca: escala de percepción del esfuerzo Borg10 (3-4).

Fuerza: ejercicios de cadena cinética cerrada y frenada, con incremento secuencial de la carga (35-45\% de su capacidad individual máxima, equivalente a un número de repeticiones que oscilan entre 8 y 12).

El control de la intensidad se hizo a través de la Escala de Borg10. Los ejercicios fueron realizados según la tolerancia de cada uno de los pacientes. 


\section{Volumen}

Resistencia cardiovascular: $10-12$ minutos

Fuerza resistencia: $8-12$ repeticiones.

\section{Duración}

70 minutos totales por semana.

Densidad: $1=3$.

\section{Ejercicios}

Resistencia cardiovascular: se utilizaron bicicletas estática y recumbent.

Fuerza: ejercicios de cadena cinética cerrada y frenada "Flexoextensión de codo, extensión hombro, press militar, flexión de codo inclinado con apoyo en pared, sentadilla”.

De la misma forma, durante esta etapa se enfatizó en los ejercicios del CORE, (incluyen tórax y abdomen), de la cintura escapular y pélvica "elevación cabeza, elevación de glúteo". Lo anterior, con el fin de lograr la máxima estabilidad central que les permitiera un mejor desempeño distal.

Se reprogramaron los momentos de alimentación e hidratación de cada uno de los pacientes en las diferentes sesiones.

Etapa de Acondicionamiento: (Semana 5 - 12), (24 sesiones presenciales).

Meta: incrementar la resistencia cardiovascular y al desarrollo de la fuerza muscular para alcanzar la realización de actividades funcionales que les permitiera llevar a cabo las actividades de la vida diaria de forma independiente, mejorando así las variables de Calidad de Vida. Se incluyeron otro ejercicio de equilibrio y coordinación. 


\section{Modo}

Resistencia cardiovascular: equipo de sonido.

Fuerza: mancuernas de 1, 2, 3, 4, 6 libras, Thera-band amarillo, rojo, verde, Azul, colchonetas.

\section{Intensidad}

Frecuencia cardiaca: escala de percepción del esfuerzo Borg10, debían mantenerse entre (34).

Fuerza: ejercicios de cadena cinética abierta, ejercicios isotónicos de resistencia progresiva para miembros superiores e inferiores utilizando como progresión desde brazos de palanca cortos hasta brazos de palanca largo según tolerancia (45-65\% de RM). La cantidad de estímulos por grupo muscular (patrón de movimiento).

\section{Volumen}

Resistencia cardiovascular: lograr los 15 minutos, ya fuese intervalado o continuos.

Fuerza: 12 a 15 repeticiones/estímulos.

\section{Duración}

85 a 110 minutos totales por semana.

Densidad: $1=3$.

\section{Ejercicios}

Resistencia cardiovascular: baile con desplazamientos continuos.

Fuerza: abducción horizontal, flexoextensión de hombro, elevación de pierna, caminata tijera, marcha militar.

Etapa de mantenimiento: comprendió las últimas 4 semanas 13-16. (12 sesiones presenciales). 
Meta: conservar la condición física adquirida y mantener el tiempo de ejecución hasta lograr la realización de actividades aeróbicas continúas por 30 minutos a través de movimientos con obstáculos similares a los que enfrentan los pacientes en las diferentes actividades de la vida diaria. Se incluyeron ejercicios de coordinación visopédica y visomanual. Para participar en esta etapa, los pacientes debían alcanzar una condición física que les permitiera una mayor resistencia cardiovascular y el manejo de cargas submáximas, lo cual implicaba la reducción del nivel de fatiga.

\section{Modo}

Resistencia cardiovascular: equipo de sonido.

Fuerza: mancuernas de 1, 2, 3, 4, 6 libras, Thera-band amarillo, rojo, verde, Azul, colchonetas, fitball de 30 y 55 cms, Step para aeróbicos de tres niveles.

\section{Intensidad}

Frecuencia Cardiaca Escala de percepción del esfuerzo Borg10, Debían mantenerse en (5).

Fuerza: Dentro de rangos submáximos (65-70\% de RM).

\section{Volumen}

Resistencia cardiovascular: 30 minutos continuos.

Fuerza: 12 a 15 repeticiones/estímulos, manteniendo el número de repeticiones/estímulos incrementando la carga en forma individualizada, según la tolerancia de cada uno de los pacientes participantes

Duración: 120 minutos totales por semana.

Densidad: $1=3$. 


\section{Ejercicios}

Resistencia cardiovascular: baile con desplazamientos continuos, además se realizaron ejercicios con obstáculos.

Fuerza: solo se utilizaron brazos de palanca largos "abducción pierna con flexión de rodilla, extensión de cadera con rodilla flexionada, elevación frontal de pierna rodilla flexionada y extendida, abducción y aducción de pierna en extensión”, Ejercicios de coordinación visopédica y visomanual: agarre en un solo eebote de fitball, equilibrio sobre en una sola pierna en step, pateo y detención de balón.

\section{$\underline{\text { Mediciones }}$}

Las pruebas se desarrollaron en dos días y se hicieron según protocolo en el mismo lugar, hora, durante dos semanas una antes de iniciar y la otra posterior al haber concluido la participación en el programa, así:

El 1er día se realizaron: 6MWT - handgrip - Sf-36 dejando un día intermedio.

El 2do día: sit and eeach - 30 sit to etand - SF-36. 